\title{
Wavelet Denoising Approach for Evaluation of EMG Signal in Sub 1 kHz Range Having Interference Signals
}

\author{
M. Karuna ${ }^{\# 1}$, P. Rajesh Kumar ${ }^{* 2}$, Dr.Rudra Pratap Das ${ }^{\# 3}$ Dr.M.S.S.Rukmini ${ }^{\# 4}$ \\ \# 1,2,3,4 Departmentof Electronics and Communication, \\ ${ }^{\# 1,3}$ Vignan's Institute of Information Technology, India \\ \#2 Andhra University, India \\ ${ }^{\# 4}$ Vignan's University, India \\ ${ }^{1}$ karunaleo98@gmail.com , ${ }^{2}$ rajeshauce@gmail.com, \\ 3drudrapratapdas@yahoo.com,4mssrukmini@yahoo.co.in
}

\begin{abstract}
Electromyography (EMG) signal picked up from the muscle fibers have signal in the range below $1 \mathrm{KHZ}$ coupled with some noise .The objective is to apply de-nosing by wavelet. Biomedical amplifier AD620 is used for detecting the EMG signal having the gain 4 and approach in 20 to $500 \mathrm{~Hz}$. A notch filter is used to eliminate $50 \mathrm{~Hz}$ pick up. Wave let de-nosing is used to estimate the transform coefficients of basis signals removing the noise. MATLAB is used to calculate the average power of signal. The muscle activates of normal and abnormal persons are compared in case of writer's cramp the cases of alphabets $\mathrm{A}, \mathrm{B}, \mathrm{C}$ are taken for comparison by trapezoidal integration.
\end{abstract}

Wavelet de-nosing, EMG signal, MATLAB, Biomedical amplifier AD620, notch filter, Wavelet transform

\section{INTRODUCTION}

"Electromyography (EMG) is an experimental technique as shown in fig.1, concerned with the development, recording and analysis of myoelectric signals. EMG signal [9]. Surface electrode converts the biomedical Signals into Electrical signals..Electromyography (EMG) picked up from the muscle or skin surface by suitable electrodes display a continuous chain of action potentials related to motor unit action potentials [MUAPS]. There is some noise associated with these. A group of action potentials of muscle fibers called MFAP give rise up MUAPS. Neuron signals [1-2] are superimposed on MFAP for maintaining a certain level of force, the alpha motor neuron excite the muscle fibres until these shrink [8].

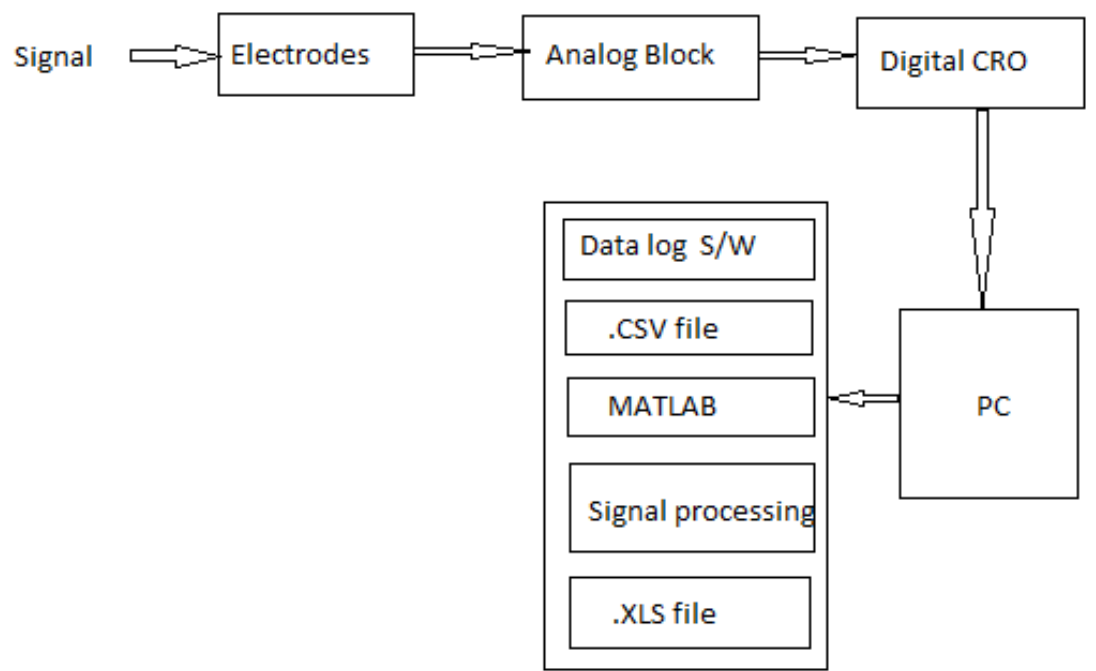

Fig.1 Block Diagram of EMG

\section{ANAlog Block}

The analog block fig.2 consists of a differential amplifier which amplifier the difference created by the feeble bio-medical signals We use AD620 a bio medical amplifier for detecting the EMG signal this is band pass filter with a band pass filter having gain 4 and range 20 to $500 \mathrm{~Hz}$. And this signal is notch filtered to eliminate $50 \mathrm{~Hz}$ pick up line voltage noise. The Band Pass filter and the notch filter which uses three 741 op amps are replaced with LM324 to have better CMRR and less circuitry. This analog signal is seen in the digital CRO and Get in to PC using USB Cable and DSWAVESETUP software. 


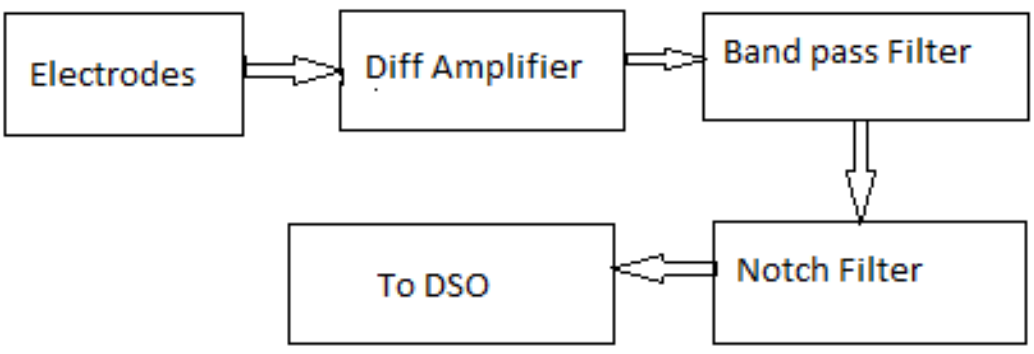

Fig.2 Analog Block

\section{III.Signal Processing}

The signal from DSO is taken and is saved as excel file. It is imported into the MATLAB [12] and the following signal processing as shown in fig. 3 steps are applied moving average filter, .Butterworth band pass filter, Butterworth band stop filter, Full wave rectification, Power Calculation, Wavelets de-nosing.
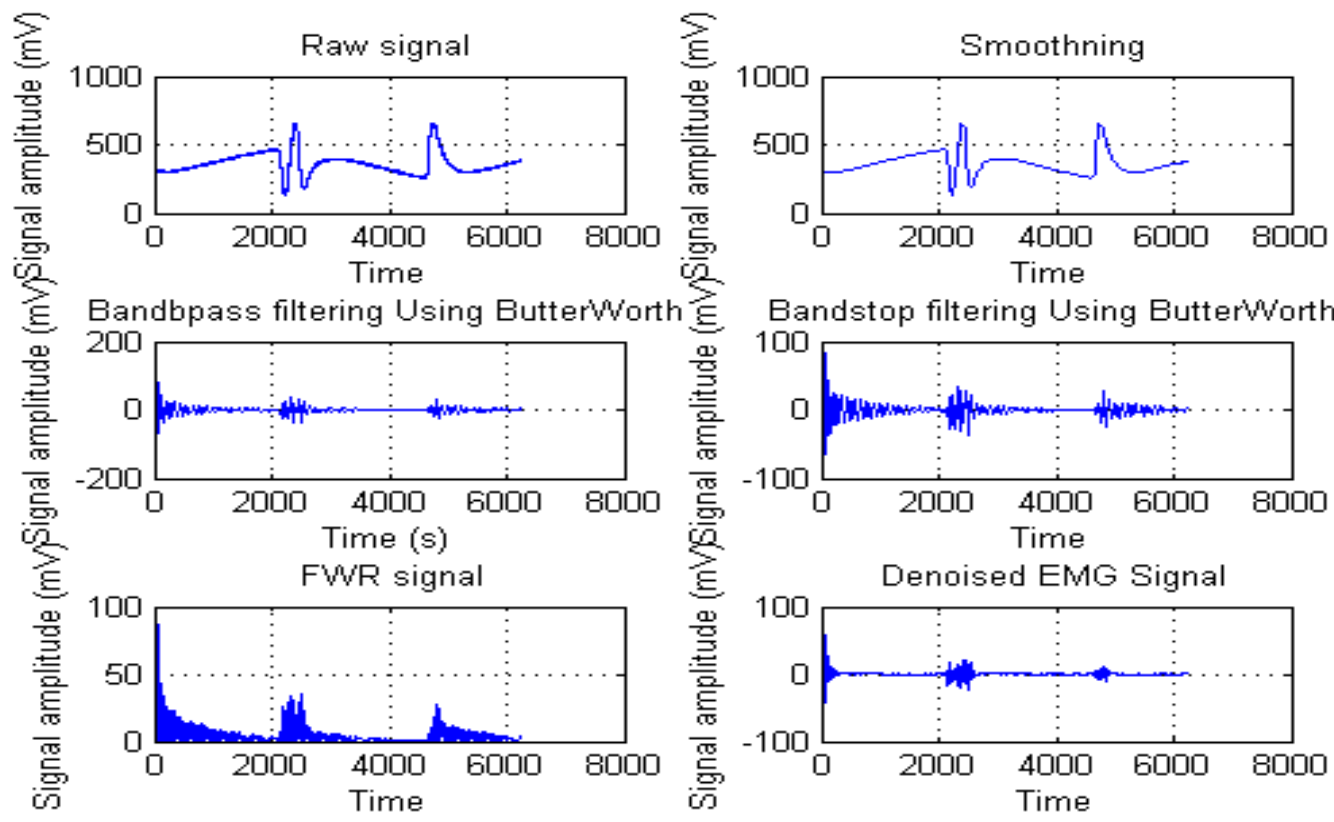

Fig 3. Signal processing

\section{IV.GETTING SIGNAL INTO MATLAB}

The saved signal which is in the form of MSEXCEL format is read into the MATLAB using xlsread command available in the MATLAB[11].

Syntax:[num,txt,raw] $=x l s r e a d(f i l e n a m e)$

[num,txt,raw] $=x$ lsread(filename,sheet)

$[$ num,txt,raw $]=x l s r e a d($ filename,range)

$[$ num,txt,raw $]=x$ lsread(filename, sheet,range)

\section{FiLTERING APPROACH}

The basic concept of filtering is to remove the unwanted frequencies and allow the pass band frequencies with the desired pass band gain .A moving average filter smoothen the signal and is used to remove the random spikes present in the signal. Here 10 point moving average filter is used to remove the spikes in signal and smoothen it. The butter worth filter gives maximally flat response using buttord function to get the optimal order of the function. The butter worth band pass filter is designed to allow frequencies from $20 \mathrm{~Hz}$ to $250 \mathrm{~Hz}$ with a pass band ripple of $2 \mathrm{~dB}$ and stop band ripple of $40 \mathrm{~dB}$. The $50 \mathrm{~Hz}$ pick up noise from the line supplies add up with the signal and it can be eliminated by using a band stop notch filter. Full wave rectification gives positive peaks and removes negative peaks present in the signal. 
The average power calculation of the EMG signal plays an important role in determining muscle activity of the muscle. By daily taking these measurements of muscle activity and saving them we can easily tell the patient condition. To calculate the muscle fatigue, we have to calculate the average power which can be calculated by using the Avg power command in MATLAB to the DSP data [5-6]. The signal power in MATLAB is first calculated by calculating power spectral density and then calculating power.

To compute the average power in particular frequency band [5], a rectangular approximation of integral of power spectral density (PSD) is used. For matrix type of signal, computation is done column wise and it is assessed if the total average power contained in one sided or two sided spectrum [10] as shown in table1

Table-1 ranges of one sided or two sided spectrum

\begin{tabular}{|c|c|c|}
\hline Type & Signal & Range \\
\hline One sided & Even & $(0, \mathrm{pi})$ \\
\hline One sided & Odd & $(0, \mathrm{pi})$ \\
\hline Two sided & Even and odd & $(0,2 \mathrm{pi})$ \\
\hline
\end{tabular}

Frequency range is two element vector of frequencies between which calculation is done in case of mismatch, the closest value is used. First frequency value is included in the calculations whereas second value is excluded.

\section{Wavelet Analysis in EMG Signal Processing}

Everywhere around us are signals that need to be analysed. Seismic tremors, human speech, engine vibrations, medical images, financial data, music, and many other types of signals have to be efficiently encoded, compressed, cleaned up, reconstructed, described, simplified, modelled, distinguished, or located[3]. Wavelet analysis is a new and promising set of tools and techniques for doing this. Wavelet analysis gives us an improved method involving a windowing technique having regions of variable dimensions. Such analysis is uses long time intervals needing exact low frequency information and shorter region of high frequency [13], in comparison with time based and short time fourier transform [3,4]:

Several families of wavelets that have proven to be especially useful are Harr, Daubechies, Biorthogonal, Coiflets, Symlets, .Morlet,. Mexican Hat,. Meyer.

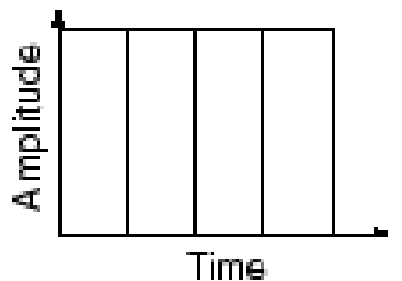

Time Domain (Shannon)

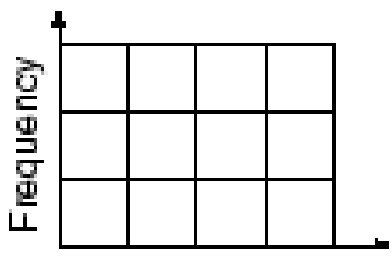

Time STFT (Gabor)
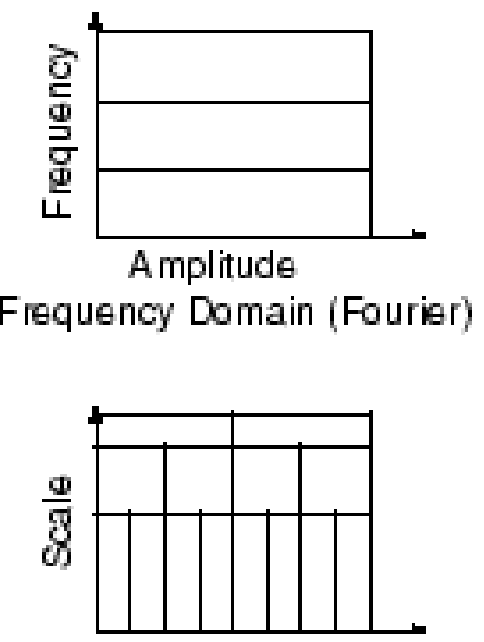

Time Wavelet Analysis

Fig.4 Wavelet Fourier analysis

The signals of EMG of are detected by skin- fixed electrodes. Muscles are known to give electric signal of the order of 0.1 to $5 \mathrm{mv}$ in developing a sensitive system we have to take into the consideration anti jamming factor, noise and interference. Generally such signals are in this same range $0-1000 \mathrm{~Hz}$ but in some cases high frequency as well as dc signals are included.

Most useful EMG signals are in the range of $50-150 \mathrm{~Hz}$ [4]. Noise is assumed to have a Gaussian distribution [5].we can represent these by

$$
f(t)=s(t)+n(t)
$$


Where $s(t), n(t)$ denotes EMG signals and White Gaussian Noise $N(0, \sigma 2)$, respectively. To do a multiresolution signal analysis for equation (1), it may be noted that the wavelet transform of signals exhibit a greater change in value when position is odd. Moreover, such coefficients for noise are uniform for all scales. When scales increase, the coefficient amplitude is less. Normally EMG signals are in large scales where as noise in small scale. So the wavelet denoising as to currently estimate the transform coefficients of basic signals after removing noise component. The conventional wavelet denoising has a threshold level which can be divided into soft and hard.

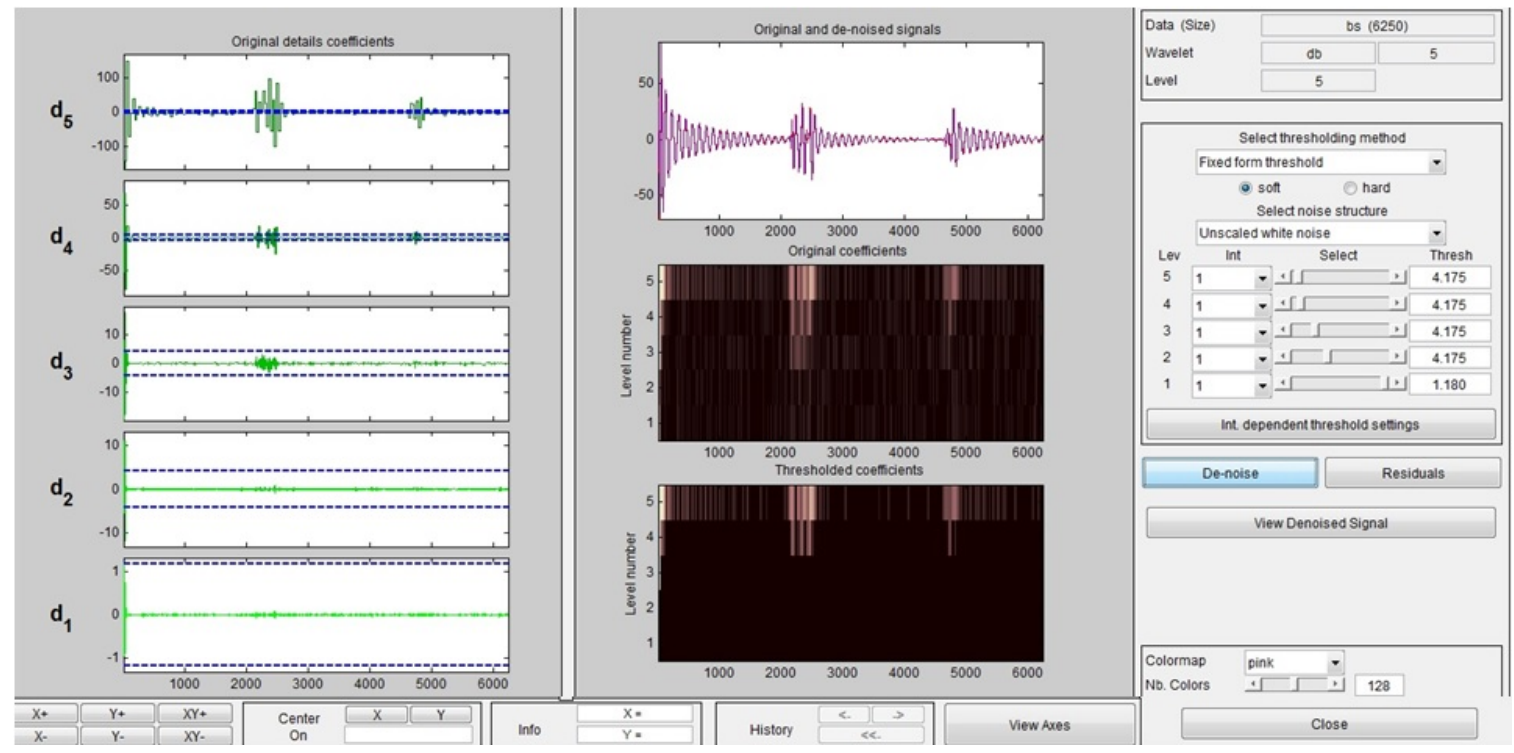

Fig.5 De-Noised EMG Signal using wavelets

\section{RESULTS}

The EMG signal is measured from the normal subject shown in fig.6 and abnormal subject shown in fig.7 who is having writer's cramp, are de-noised their muscle activities [7] while writing alphabets A, B, C and also compared both normal and abnormal shown in fig.8.

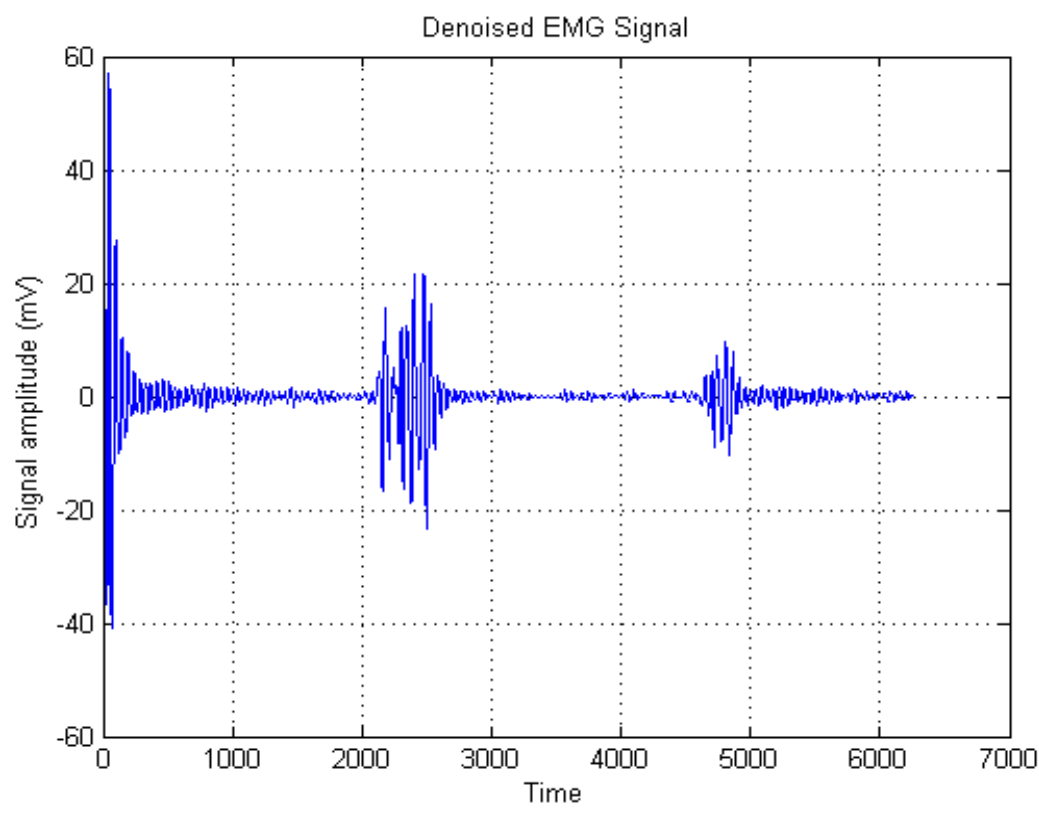

Fig.6 De-Noised EMG Signal for Normal Subject 


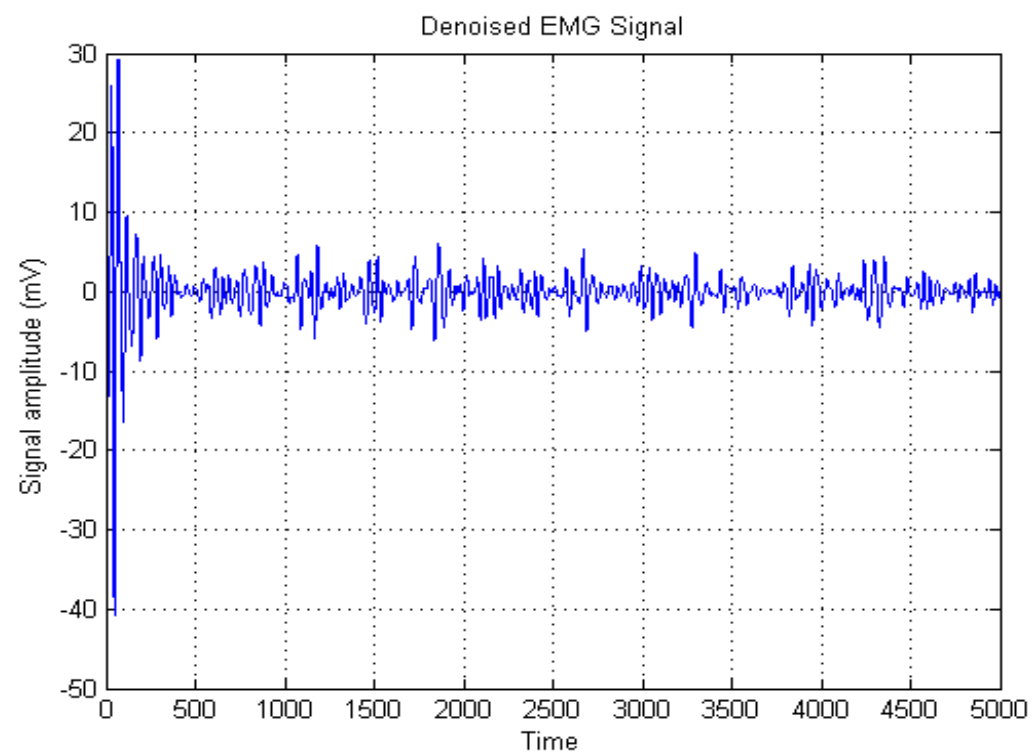

Fig.7 De-Noised EMG Signal for Abnormal Subject

The observational values of normal and abnormal subjects are shown in table-2 while hand writing.

Table-2 MUAPs for normal and abnormal persons

\begin{tabular}{|c|c|c|}
\hline Sl.no & Max Amplitude Range & Observation \\
\hline 1 & -180 to $200 \mathrm{mV}$ & Normal \\
\hline 2 & -5 to $4 \mathrm{mV}$ & Abnormal \\
\hline
\end{tabular}

$\mathrm{Z}=\operatorname{cumtrapz}(\mathrm{X}, \mathrm{Y})$ computes the cumulative integral of $\mathrm{Y}$ with respect to $\mathrm{X}$ using trapezoidal integration.

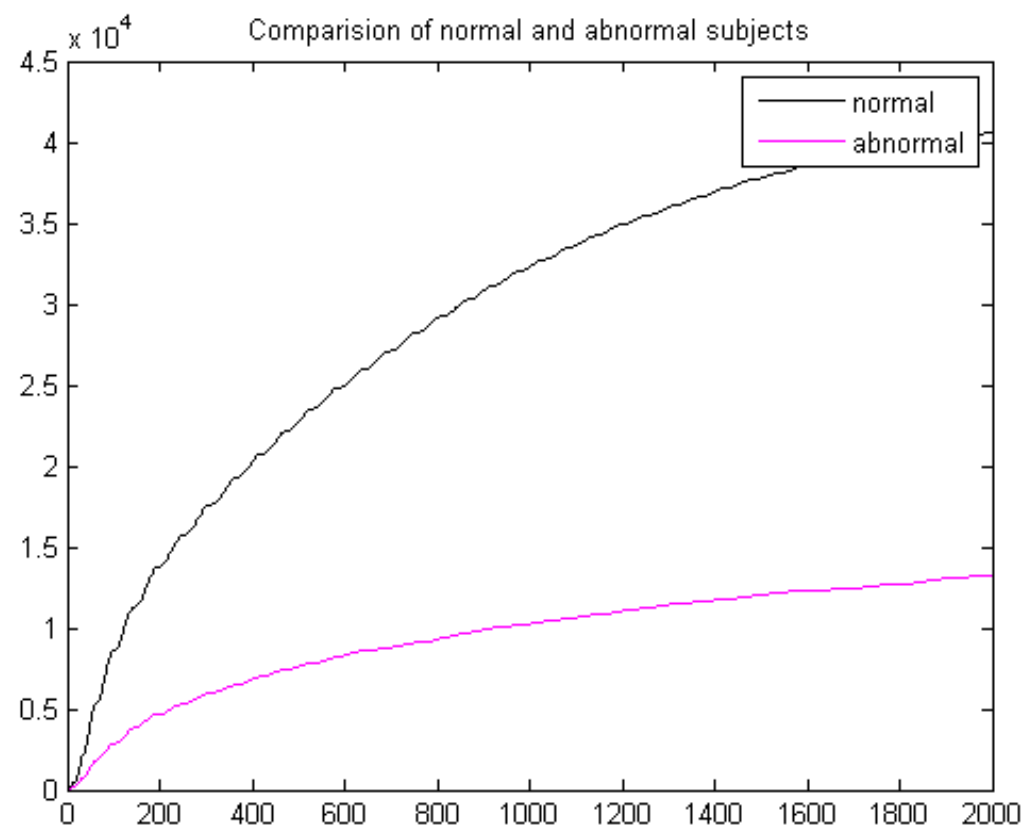

Fig.8 Comparison of normal and abnormal persons while hand writing

\section{CONCLUSION}

Finally for proposed system effective signal detection has been obtained but with some noise more noise redundant methods are to be used to get pure signal .An effective data base with high recognition has to be implemented. 


\section{REFERENCES}

[1] S. Karlsson, J. Yu, M. Akay, "Time-frequency analysis of myoelectric signals during dynamic contractions: a comparative study", IEEETrans. Biomed. Eng., vol. 47, no. 2, Feb 2000..

[2] P. D. Welch, "The Use of Fast Fourier Transform for the Estimation of Power Spectra: A Method Based on Time Averaging Over Short, Modified Periodograms", IEEE Trans. on Audio Electro acoustics, vol. A-15, pp. 70-73, June 1967.

[3] ShahjahanShahid*, Jacqueline Walker, Member, IEEE, Gerard M. Lyons, Ciaran A. Byrne, and AnandVishwanath Nene" Application of Higher Order Statistics Techniques to Signals to Characterize the Motor Unit Action Potential" VOL. 52, NO. 7, JULY 2005

[4] C. L. Nikias and M. R. Raghuveer, "Bispectrum estimation: a digital signal processing framework," Proc. IEEE, vol. 75, no. 7, pp. 869-891, Jul. 1987.

[5] Yin Chen, Jiahai Liu, "Study on denoising of surface EMG signals," Computer Era, vol. 26, No. 6, pp. 22-24, 2008.

[6] Gilat, Amos (2004). MATLAB: An Introduction with Applications 2nd Edition. John Wiley \& Sons. ISBN 978-0-471-69420-5.

[7] A. A. Rodriquez and J. C. Agre, "Electro physiologic study of the quadriceps muscles during fatiguing exercise and recovery: a comparison of symptomatic and asymptomaticpostpolio patients and controls," Arch.Phys. Med. Rehab., vol. 72, pp. 993-997, 1991.

[8] Kleissen RFM, Buurke JH, Harlaar J, Zilvold G. Electromyography in the biomechanical analysis of human movement and its clinical application. Gait Posture 1998; 8(2):143-158..

[9] Cram JR, Kasman GS, Holtz J. Introduction to Surface Electromyography. Aspen Publishers Inc.; Gaithersburg, Maryland, 1998.

[10] Reaz MB, Hussain MS, Mohd-Yasin F. Techniques of EMG signal analysis: detection, processing, classification and applications. BiolProced Online. 2006;8(1):11-35. doi: 10.1251/bpo115.

[11] Semmlow, John. L., "Biosignal and Bimedical Image Processing MATLAB-BASED APPLICATIONS," Rebert Wood Johnson Medical School, New Brunswick, New Jersey, US.

[12] T. David Mewette, N. Homer, J. R. Karen, -Removing Power Line Noise from Recorded EMGll, Proceedings of the 23rd annual international conference, pp 2190-2193, Oct 25-28, Istanbul, Turkey.

[13] P. W. Mark, -Wavelet-based noise removal for biomechanical signals: A comparative studyll, IEEE Trans. on biomedical engineering, vol 47, no. 2, pp. 360-360.

\section{AUTHOR PROFILE}

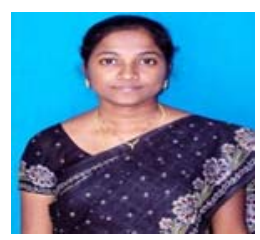

Mrs. M. Karuna working as Associate Professor in the Department of Electronics and Communication Engineering, Vignan's Institute of Information Technology, Visakhapatnam. Interested fields are Biomedical signal processing, Biomedical Instrumentation.

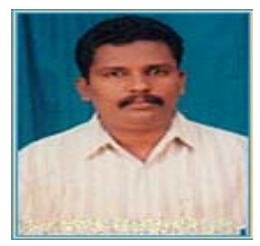

Dr. Pullakura Rajesh Kumar M.E., Ph.D.Working as professor in Department of Electronics \& Communication Engineering, College of Engineering, Andhra University, and Visakhapatnam.Interested fields are Radar Signal Processing, Bio-Medical Signal Processing.

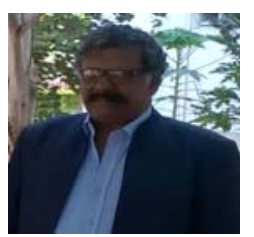

Dr. Rudra Pratap Das Ph.D.Working as professor in Department of Electronics \& Communication Engineering, Vignan's Institute of Information Technology, and Visakhapatnam. Interested fields are, Bio-Medical Signal Processing, Fuzzy logic and Neural Networks.

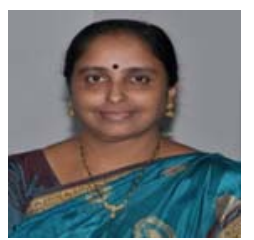

Dr. M.S.S.Rukmini working as professor in the department of Electronics and Communication Engineering from Vignan's University, Vadlamudi. Interested fields are, Wireless Communications, Embedded systems, Bio Signal processing. 